\title{
RESEARCH
}

Open Access

\section{GREM1 is associated with metastasis and predicts poor prognosis in ER-negative breast cancer patients}

Ulrike Neckmann ${ }^{1,2,3 \dagger}$, Camilla Wolowczyk ${ }^{1,2,3 \dagger}$, Martina Hall ${ }^{4,5}$, Eivind Almaas ${ }^{4,5}$, Jiang Ren ${ }^{6}$, Sen Zhao ${ }^{7}$, Bjarne Johannessen ${ }^{7}$, Rolf I. Skotheim ${ }^{7,8}$, Geir Bjørkøy ${ }^{1,2}$, Peter ten Dijke ${ }^{6}$ and Toril Holien ${ }^{9,10^{*}}$ (D)

\begin{abstract}
Background: In breast cancer, activation of bone morphogenetic protein (BMP) signaling and elevated levels of BMP-antagonists have been linked to tumor progression and metastasis. However, the simultaneous upregulation of BMPs and their antagonist, and the fact that both promote tumor aggressiveness seems contradictory and is not fully understood.

Methods: We analyzed the transcriptomes of the metastatic $66 \mathrm{cl} 4$ and the non-metastatic $67 \mathrm{NR}$ cell lines of the 4T1 mouse mammary tumor model to search for factors that promote metastasis. CRISPR/Cas9 gene editing was used for mechanistic studies in the same cell lines. Furthermore, we analyzed gene expression patterns in human breast cancer biopsies obtained from public datasets to evaluate co-expression and possible relations to clinical outcome.

Results: We found that mRNA levels of the BMP-antagonist Grem1, encoding gremlin1, and the ligand Bmp4 were both significantly upregulated in cells and primary tumors of $66 \mathrm{cl} 4$ compared to $67 \mathrm{NR}$. Depletion of gremlin 1 in $66 \mathrm{cl} 4$ could impair metastasis to the lungs in this model. Furthermore, we found that expression of Grem 1 correlated with upregulation of several stem cell markers in $66 \mathrm{cl} 4$ cells compared to 67NR cells. Both in the mouse model and in patients, expression of GREM1 associated with extracellular matrix organization, and formation, biosynthesis and modification of collagen. Importantly, high expression of GREM1 predicted poor prognosis in estrogen receptor negative breast cancer patients. Analyses of large patient cohorts revealed that amplification of genes encoding BMP-antagonists and elevation of the corresponding transcripts is evident in biopsies from more than half of the patients and much more frequent for the secreted BMP-antagonists than the intracellular inhibitors of SMAD signaling.
\end{abstract}

Conclusion: In conclusion, our results show that GREM1 is associated with metastasis and predicts poor prognosis in ER-negative breast cancer patients. Gremlin1 could represent a novel target for therapy.

Keywords: 4T1 model, 66cl4, 67NR, BMP4, GREM1, Metastasis, Gremlin 1

\footnotetext{
* Correspondence: toril.holien@ntnu.no

Ulrike Neckmann and Camilla Wolowczyk are Co-first author

${ }^{9}$ Department of Clinical and Molecular Medicine (IKOM), NTNU, Gastro

Center, Prinsesse Kristinas gt 1, 7030 Trondheim, Norway

${ }^{10}$ Department of Hematology, St. Olavs Hospital, Trondheim, Norway

Full list of author information is available at the end of the article
}

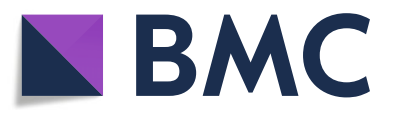

(c) The Author(s). 2019 Open Access This article is distributed under the terms of the Creative Commons Attribution 4.0 International License (http://creativecommons.org/licenses/by/4.0/), which permits unrestricted use, distribution, and reproduction in any medium, provided you give appropriate credit to the original author(s) and the source, provide a link to the Creative Commons license, and indicate if changes were made. The Creative Commons Public Domain Dedication waiver (http://creativecommons.org/publicdomain/zero/1.0/) applies to the data made available in this article, unless otherwise stated. 


\section{Background}

Members of the transforming growth factor $\beta$ (TGF- $\beta$ ) family, including the TGF- $\beta \mathrm{s}$, activins, nodal, bone morphogenetic proteins (BMPs), and growth and differentiation factors (GDFs), play important roles in tumor progression and formation of metastases [1]. However, these signaling molecules have pleiotropic effects in cancer, as seen with TGF- $\beta$ signaling, which has been linked to both tumor suppression and tumor promotion depending on the tumor stage [2, 3]. After binding of the TGF- $\beta$ s to transmembrane receptors, SMAD2 and SMAD3 become phosphorylated and bind to SMAD4; subsequent translocation of this complex to the nucleus result in the expression of TGF- $\beta$ controlled genes. In contrast, BMP signaling primarily occurs via SMAD1/ $5 / 8$ instead of SMAD2/3. Receptor activation by TGF- $\beta$ family ligands can also result in induction of nonSMAD pathways, including mitogen-activated protein kinases (MAPK), and PI3K-Akt-mTOR pathways [4]. Like TGF- $\beta$ s, BMPs have been implicated in both tumor suppression and tumor progression [5]. Understanding the role of TGF- $\beta$ family members in cancer and the development of TGF- $\beta$ targeted therapies have been hampered by the complex interplay of these signaling molecules with intracellular SMAD-inhibitors and extracellular BMP-antagonists. The expression of intracellular inhibitory SMADs, i.e. SMAD6 and SMAD7, is induced in response to several TGF- $\beta$ ligands and form a negative feedback loop. Extracellular BMP-antagonists, like noggin, gremlin1, Dand5, and follistatin, bind to BMPs and prevent binding of the BMPs to their respective receptor [6, 7]. Like BMPs and TGF- $\beta$ s, also BMP-antagonists can have a tumorpromoting role. For instance, Dand5, also known as Coco, facilitated formation of lung metastases in the 4T1 mammary tumor model by blocking lung-derived BMPs [8], whereas gremlin1 promoted stem cell maintenance in both glioma [9] and colorectal cancer [10].

Metastasis is a complex multi-step process, including local invasion, intravasation of tumor cells into the circulation, tumor cell dissemination, extravasation at metastatic site, survival at secondary site, and finally proliferation and formation of metastases [11]. To metastasize, cancer cells of epithelial origin have to acquire new properties such as increased motility, anchorage-independent growth and the ability to adapt to a foreign microenvironment. Only cancer cells that display a high degree of phenotypic plasticity and thus are able to adapt to stressful conditions and changing environments, will survive. For example, migratory tumor cells require stem cell-like properties including a low differentiation status [12].

The immunocompetent mouse model for metastatic mammary carcinoma called 4T1 consists of several cell lines isolated from the same spontaneously formed tumor $[13,14]$. All the cell lines form primary tumors when injected into the fat pad of BALB/c mice, but they differ widely in their ability to form distant metastasis. Here, we focused on $67 \mathrm{NR}$, which does not metastasize, and $66 \mathrm{cl} 4$, which metastasizes to the lungs. We hypothesized that their different metastatic propensity relates to differences in the secretome of the two cell lines, since cancer cell-derived factors influence the phenotype of the cancer cells and/or the stroma cells. We therefore performed transcriptome analysis of $66 \mathrm{cl} 4$ and $67 \mathrm{NR}$ cells from in vitro culture and grown as tumors in BALB/c nude mice and focused on differentially expressed mRNAs encoding secreted proteins. Expression of the BMP-antagonist Grem1 was significantly upregulated in 66 cl4 compared to 67NR both in cell culture and primary tumors. Gremlin1 binds and inhibits BMP2, BMP4, and BMP7 [15], which play important roles during cellular differentiation [1]. In line with the elevated expression of Grem1 in 66cl4, several stem cell markers were upregulated in the $66 \mathrm{cl} 4$ cells. Interestingly, compared to the non-metastatic 67NR cells, the metastatic 66cl4 cells also expressed significantly elevated levels of Bmp4. Consistent with a role of these secreted proteins in metastasis, we found that high GREM1 expression correlated with reduced relapse-free survival (RFS) in estrogen receptor (ER)negative breast cancer patients and that the association with poor prognosis remains, even with elevated coexpression of a BMP in the tumor.

\section{Methods}

For information about Transcriptome analysis; Quantitative real-time PCR; Immunoblotting; Harvest of conditioned medium; ELISA; In vitro extravasation assay; Cell proliferation assay; Soft-agar assay; Flow cytometry; see Additional file 1: Supplementary Methods.

\section{Cell culture and generation of stable cell lines}

The mouse mammary tumor cell lines $67 \mathrm{NR}$ and $66 \mathrm{cl} 4$ cell lines were obtained from Barbara Ann Karmanos Cancer Institute, Detroit, MI, USA. The cells were cultured in DMEM (Lonza, BioWhittaker, BE12- 604F) supplemented with $10 \%$ fetal calf serum (FCS, Thermo Fischer Scientific, \#10270-106), 2 mM L-Glutamine (Lonza Group, De-17-605E) and $50 \mathrm{U} / \mathrm{ml}$ penicillinstreptomycin (ThermoFischer Scientific, Gibco, \#15070-063). Primary umbilical vein endothelial cells, normal, human (HUVEC) were obtained from ATCC (ATCC-PCS-100-010). The cells were cultured in Medium 200 (Gibco, M200500) supplemented with low serum growth supplement (LSGS) (Gibco, S00310). All cells were incubated at $37^{\circ} \mathrm{C}$ with $5 \% \mathrm{CO}_{2}$. 
CRISPR/Cas9-GREM1 knockouts and CRISPR/Cas9 vector control cell lines were generated by viral transduction. The mouse lentiviral particles were purchased from Sigma Aldrich (CRISPR/Cas9-GREM1 Mm0000325632 and Mm0000325634; CRISPR/Cas9-NT, CRISPR12V1EA). $6 \mathrm{~h}$ after seeding, 66cl4 cells were infected with lentiviral particles (MOI 0.1) in medium containing hexadimethrine bromide $(8 \mu \mathrm{g} / \mathrm{ml})$. After $24 \mathrm{~h}$ cells were split 1:17. Starting $48 \mathrm{~h}$ after infection, cells were selected with puromycin $(3.25 \mu \mathrm{g} / \mathrm{ml})$ for 1 week. The selection medium was replaced every $2-3$ days. Single cell colonies were picked using cloning cylinders and tested for gremlin1 expression levels by western blot. The CRISPR/Cas9 NT_ mix was generated by trypsinizing a plate containing thousands of puromycin resistant colonies.

\section{Orthotopic mouse tumors and in vivo lung colonization assay}

For all experiments, female mice (8-11 weeks old, Janvier Labs, France) were used. For orthotopic tumors, mice were anaesthetized and injected with $5 \times 10^{5}$ viable cells resuspended in PBS into the fourth mammary fat pad. When palpable, tumor size was measured twice weekly using electronic calipers. Tumor volume was calculated: $V_{T}=\left(\right.$ length $x$ width $\left.^{2}\right) / 2$. NT controls and gremlin1 depleted cell lines were injected into nude mice (BALB/cAnNRj-Foxn $\left.1^{n u / n u}\right), n=10$ per cell line. Mice were sacrificed after 21 days, unless otherwise stated. Weight of primary tumors and lungs was recorded. For the in vivo lung colonization assay female mice were anaesthetized and injected with $5 \times 10^{5}$ cells $/ 100 \mu \mathrm{l}$ PBS in the lateral tail vein. Mice were monitored daily and sacrificed 15 days after injection. Entire lungs were weighed.

\section{Zebrafish xenograft model}

The transgenic fish line $\operatorname{Tg}(f l i 1: G F P)$ was used $[16,17]$. The tumor cell lines were fluorescently labelled with mCherry using plv-mCherry lentiviruses. G418 was used as selection marker. Embryo preparation and tumor cell implantation was done as previously described [18]. Briefly, $\operatorname{Tg}(f l i 1: G F P)$ zebrafish embryos were dechorionated 2 days post fertilization. Single cell suspensions of 66cl4 wildtype cells and variants were re-suspended in PBS and kept at $4{ }^{\circ} \mathrm{C}$ until transplantation. Cell suspensions were loaded into borosilicate glass capillary needles ( $1 \mathrm{~mm}$ O.D. $\times 0.78 \mathrm{~mm}$ I.D.; Harvard Apparatus) and injected into the duct of Cuvier (DoC) using a Pneumatic Picopump and a manipulator (WPI, Stevenage, UK). After injection of approximately 400 cells, zebrafish embryos were maintained at $33^{\circ} \mathrm{C}$. Invasive clusters were quantified 6 days post-implantation (dpi). Zebrafish embryos were fixated with $4 \%$ paraformaldehyde at $4{ }^{\circ} \mathrm{C}$ overnight and were imaged in PBS using a Leica SP5
STED confocal microscope. Confocal stacks were processed for maximum intensity projections with Image J. Brightness and contrast of images were adjusted with Adobe Photoshop CS6. For each cell line, at least fifty embryos were analyzed.

\section{Gene co-expression network analysis}

RNA-seq data were downloaded from the study by Ciriello et al. in Cell, 2015 [19] from The Cancer Genome Atlas (TCGA; http://cancergenome.nih.gov). The cohort consists of 817 cases of breast cancer with 17,214 genes, normalized within samples to the median gene expression. Due to missing values, only 421 cases and 16,749 genes were available for download and analysis. To assess the association between GREM1 and other genes, an ego-centric network with GREM1 in the center was created using sample Pearson correlation. The sample Pearson correlation represents the similarity between GREM1 and the corresponding gene, in terms of linear correlation. The correlations range from -1 to 1 , where a value of 1 represents a total positive linear correlation, e.g. similar expression values of GREM1 and the corresponding gene, -1 represents a total negative linear correlation, e.g. opposite expression values of GREM1 and the corresponding gene. A value near zero represents no linear correlation, e.g. no linear association between the expression values of GREM1 and the corresponding gene.

\section{KM plotter}

KM plotter (http://kmplot.com/analysis/) is an online tool for examining prognostic markers in breast cancer subtypes, which utilizes data from multiple cDNA microarray experiments [20, 21]. Survival analysis (RFS or OS) was done using the KM plotter database 2017 version. High and low expression were defined as above (hazard ratio $(\mathrm{HR})>1.2, p$-value $<0.05)$ and below $(\mathrm{HR}<0.83$, p-value $<0.05)$ median, except for the OS analysis where best cutoff was used.

\section{cBioPortal}

cBioPortal (http://www.cbioportal.org/) is an openaccess database that allows visualization and analysis of large-scale cancer genomics data sets [22, 23]. Our analyses utilize the OncoPrints visualization to identify genomic alterations, including somatic mutations, mRNA expression and amplifications across a set of cases. This visualization shows the genes as rows, while individual cases are shown as columns. For this analysis we used the TCGA Provisional data set for invasive breast carcinoma, and selected mutations, putative copy-number alterations and mRNA expression as genomic profiles. 


\section{Gene enrichment analysis}

Gene enrichment analyses were done with the online tools Enrichr (http://amp.pharm.mssm.edu/Enrichr/) [24, 25] for the $66 \mathrm{cl} 4 / 67 \mathrm{NR}$ transcriptome data (Fig. 2b) and Reactome (https://reactome.org/) [26] for the TCGA cohort (Fig. 3b).

\section{Co-expression analysis}

Gene co-expression analysis was performed using the online gene co-expression search engine SEEK (SearchBased Exploration of Expression Compendium [Human]) (http://seek.princeton.edu/) [27] searching 331 breast cancer patient data sets. In addition, both Expression Atlas (https://www.ebi.ac.uk/gxa) [28] and CCLE (Broad Institute Cancer Cell Line Encyclopedia, https:// portals.broadinstitute.org/ccle) [29] was used for gene expression analysis in human breast cancer cell lines.

\section{Statistics}

Statistical analyses were performed in GraphPad Prism 7. Values are expressed as mean \pm standard deviation (SD), or mean \pm standard error of the mean (SEM). Statistical analyses were performed by paired 2-tailed Student's t-test after log-transformation $\left({ }^{*} 0.01<P<0.05\right.$, **0.001 $<P<0.01$, *** $P<0.001)$.

\section{Results}

Grem 1 is highly expressed in the metastatic $66 \mathrm{cl} 4$ cells and elevated GREM1 in tumor biopsies correlates with reduced relapse-free survival in patients

Cancer cells' ability to metastasize may be affected by factors that are secreted by the cancer cells (autocrine) or by factors secreted by cells in the tumor microenvironment (TME) (paracrine). In search of factors that promote metastasis, we therefore compared the expression of genes affecting the TME in the non-metastatic 67NR and the metastatic $66 \mathrm{cl} 4$ cells from the $4 \mathrm{~T} 1$ mouse model. Using RNA-sequencing (RNA-seq), we compared the transcriptomes of cells grown in culture and isolated from primary tumors in mice and we focused on transcripts encoding matrisome-associated extracellular matrix (ECM) affiliated proteins (164 genes) and secreted factors (363 genes) as defined by Naba et al. [30]. We detected 220 of these transcripts in either cell cultures or primary tumors of $66 \mathrm{cl} 4$ or $67 \mathrm{NR}$ (cut-off: 1 fragment per kilobase of mRNA per million mapped reads [FPKM]). Of these, 28 genes were significantly overexpressed in both cells and primary tumors in $66 \mathrm{cl} 4$ compared to $67 \mathrm{NR}$ (cut-off: fold-change $\geq 1.5, p$-value $\leq 0.05$ ) (Fig. 1a). We then analyzed if increased mRNA expression levels of any of these 28 secreted factors correlated with reduced RFS in breast cancer patients. Using KM plotter [20, 21], we found that high expression of GREM1 correlated with RFS in all breast cancer cases $\left(\mathrm{HR}=1.32(1.18-1.47)\right.$, $\mathrm{p}$-value $\left.=6.9 \mathrm{e}^{-07}\right)$ and $\mathrm{ER}$ - negative subtype $\quad(\mathrm{HR}=1.51 \quad(1.2-1.9), \quad \mathrm{p}$-value $=$ 0.00037), whereas in the ER-positive subtype there was a significant correlation with RFS, but the HR was just below cut-off $(\mathrm{HR}=1.19 \quad(1.01-1.4), \quad \mathrm{p}$-value $=0.035)$ (Fig. 1b). Of note, a poorer overall survival (OS) was also seen in the patients with the highest levels of GREM1 mRNA in tumor biopsies (Additional file 2: Figure S1). We confirmed overexpression of Grem1 mRNA levels in 66cl4 by quantitative PCR (Q-PCR) (Fig. 1c). Moreover, western blot and ELISA analysis showed that gremlin1 was also highly expressed on protein level and was secreted by $66 \mathrm{cl} 4$, but not $67 \mathrm{NR}$ (Fig. 1d and e). Surprisingly, $B m p 4$, which is one of the three preferred ligands that gremlin 1 binds and inhibits, was also among the 28 upregulated secreted factors in the metastatic $66 \mathrm{cl} 4$ cells compared to the non-metastatic 67NR cells (Fig. 1a). Overexpression of Bmp4 in 66cl4 was validated by QPCR (Fig. 1f) and western blot (Fig. 1g). However, besides GREM1, high mRNA levels of BMP4 or any of the other 28 secreted factors that were upregulated in the metastatic $66 \mathrm{cl} 4$ cells did not correlate with reduced RFS (Additional file 3: Table S1). On the contrary, a reduced RFS correlated with low mRNA levels for some of these genes. We therefore wanted to study the role of gremlin1 in aggressive tumor development and metastasis more carefully.

\section{Genetic alterations in BMP-antagonists are common in invasive breast cancer}

Increased gremlin 1 activity has been shown to promote epithelial-to-mesenchymal transition (EMT) and maintenance of stem cell-like properties in glioma and colorectal cancer cells $[9,10,31]$. In addition, other BMP-antagonists, including Dand5 [8] and noggin [32], have been linked to tumor progression and metastasis. The RNA-seq analysis revealed that also other BMPantagonists were expressed (>1 FPKM) and significantly upregulated either in cell culture or primary tumors in 66cl4 compared to 67NR (Fig. 2a and Additional file 4: Table S2). Furthermore, the RNA-seq analysis showed a significant upregulation of the two inhibitory SMADs, Smad6 and Smad7, in 66cl4 (Fig. 2a). Thus, BMP activity may be more repressed in the metastatic $66 \mathrm{cl} 4$ cells than the non-metastatic 67NR cells, both by extracellular antagonists and intracellular inhibitors. Consistently, we found increased SMAD4-related gene expression in 67NR by gene enrichment analysis (Enrichr [24, 25]) of the 1252 transcripts that were significantly upregulated in the $67 \mathrm{NR}$ cells and primary tumors compared with $66 \mathrm{cl} 4$ (Fig. 2b). To assess if our finding in the mouse model could have clinical relevance, we searched the TCGA provisional data set for invasive breast carcinoma in the online tool cBioPortal $[22,23]$ for gene alterations in BMP-antagonists and SMADs in invasive breast 


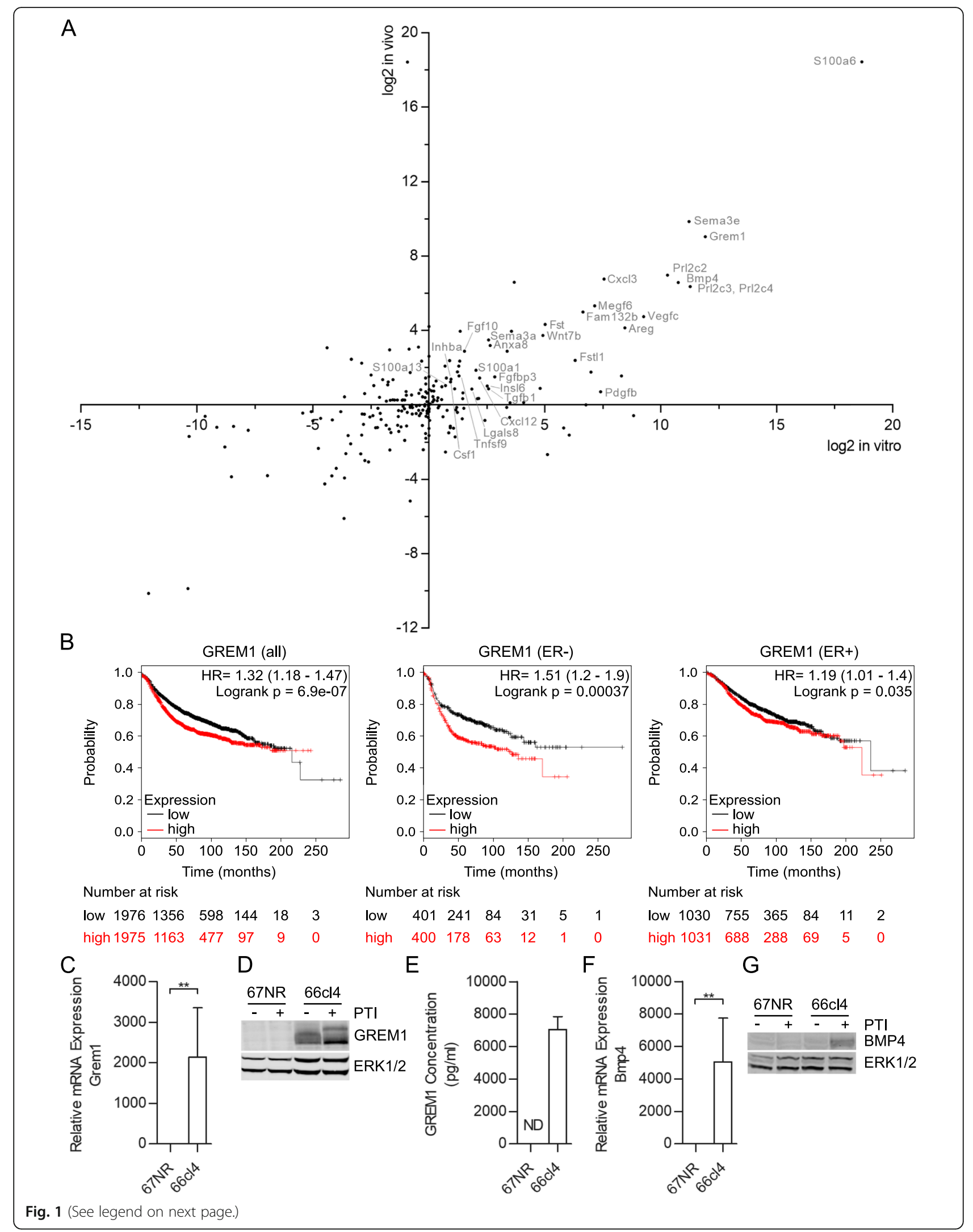


(See figure on previous page.)

Fig. 1 Grem 1 is highly expressed in 66cl4 and GREM1 correlates with RFS in breast cancer patients. (a) Scatter plot of in vivo versus in vitro differential expression of mRNAs encoding secreted proteins in $66 \mathrm{cl} 4$ and $67 \mathrm{NR}$. A positive number indicates higher expression in $66 \mathrm{cl} 4$, whereas a negative number indicates higher expression in 67NR. The 28 factors were significantly overexpressed in both 66cl4 cells and 66cl4 primary tumors (cut-off: fold-change $\geq 1.5, p$-value $\leq 0.05$ ) are indicated by name. (b) Relationship between GREM1 gene expression and RFS in breast cancer patients using KM plotter. High and low expression were defined as above and below median. (c) Q-PCR analysis of Grem1 mRNA expression in $67 \mathrm{NR}$ and $66 \mathrm{cl} 4 \mathrm{cell}$ lines in vitro $(n=3)$. Fold change is relative to Actb and Tbp. Results are shown as mean \pm SEM. Student's t-test, ${ }^{* *} 0.001<P<0.01$. (d) Representative gremlin1 immunoblot $(n=4)$ of whole lysates of the $67 \mathrm{NR}$ and $66 \mathrm{cl} 4$ cell lines without or with PTI for $6 \mathrm{~h}$. (e) Levels of gremlin1 in conditioned medium from $67 \mathrm{NR}$ and $66 \mathrm{cl} 4(\mathrm{n}=3)$ measured by ELISA. Results are shown as mean \pm SEM. (f) Q-PCR analysis of Bmp4 mRNA expression in 67NR and 66cl4 cell lines in vitro $(n=3)$. Fold change is relative to Actb and Tbp. Results are shown as mean \pm SEM. Student's t-test, ${ }^{* *} 0.001<P<0.01$. (g) Representative BMP4 immunoblot $(n=4)$ of whole lysates of the $67 \mathrm{NR}$ and $66 \mathrm{cl} 4$ cell lines without or with PTI for $6 \mathrm{~h}$

cancer patient samples. Since there is no complete list of existing BMP-antagonists, we chose to include the most recognized extracellular antagonists, as well as the two membrane-bound antagonists, BAMBI and CRIM1, in this comparison [7, 33]. Interestingly, we found genetic changes (mutations, amplifications, deletions or altered mRNA levels) in the selected genes in as much as $62 \%$ (53\% if the SMADs are omitted) of the 960 samples analyzed (Fig. 2c). The data indicate that deregulation of BMP-antagonists in patients can happen via different mechanisms. For instance, GREM2 is commonly amplified in breast cancer patients, indicating that elevated levels of GREM2 origins from the cancer cells and not the surrounding tissue. In comparison, increased levels of GREM1 mRNA (found in 6\% of the tumor biopsies) are rarely caused by amplifications. Thus, elevated GREM1 in breast cancer tumors may either originate from normal cells in the tumor microenvironment or from the tumor cells themselves.

We then asked if any of the extracellular antagonists besides GREM1, or inhibitory SMADs, could predict prognosis of breast cancer patients. In all breast cancer cases, elevated levels of GREM1 and SMAD6 correlated with poor prognosis (Fig. 1b and Additional file 5: Table S3). In contrast, low mRNA levels of six BMP-antagonists (CER1, CHRD, CHRDL1, CRIM1, DAND5, and FST) correlated with reduced RFS. Moreover, low mRNA levels of BMPER, CHRDL1, CRIM1 and SOSTDC1 correlated with reduced RFS in ER-positive breast cancer patients and high mRNA levels of CRIM1, GREM1 and SMAD6 correlated with reduced RFS in ER-negative breast cancer patients. In summary, these data indicate that in contrast to other BMP-antagonists, high GREM1 expression is particularly relevant for aggressive tumor development in breast cancer patients.

\section{Gene co-expression network analysis identifies genes involved in collagen formation and ECM organization associated with GREM1}

A feature of metastatic cells is the ability to undergo EMT, a process where the tumor cells lose their epithelial characteristics and gain a mesenchymal phenotype. This phenotype is associated with expression of genes that modulate ECM and allows for tumor cell motility and invasion [34]. We hypothesized that gremlin1 is involved in modulation of ECM and that GREM1 expression correlates with expression of genes known to be involved in this process in patients. To test this, we performed a co-expression network analysis of RNA-seq data of 421 breast cancer cases from TCGA (study by Ciriello et al., Cell, 2015) [19]. The correlation values between the 16,749 genes tested and GREM1 were in the range $-0.32-0.72$. An ego-centric network was made by the 50 top-scoring transcripts with positive correlations [range 0.59-0.72], meaning that these genes and GREM1 have similar expression patterns across samples (Fig. 3a). The location of the genes in the network represents the strength of association, where the ones closest to GREM1 correlate best. Gene enrichment analysis of the same 50 top-scoring genes using the Reactome pathway database [26] demonstrated that genes involved in ECM organization, collagen formation, and collagen biosynthesis and modification were enriched (Fig. 3b). These results were confirmed by the gene co-expression search engine SEEK [27], searching 331 breast cancer data sets (Additional file 6: Table S4 and Additional file 7: Table S5). Combined, the data suggest that gremlin1 may be involved in ECM modification, which favor tumor cell migration and intravasation, leading to increased metastasis and consequently poor prognosis.

\section{Grem 1 depletion might impair $66 \mathrm{cl4}$ 's ability to form metastases in the lungs}

To determine if gremlin1 expression is important for tumor formation and progression, we used CRISPR/ Cas9 to generate gremlin1 depleted $66 \mathrm{cl} 4$ cells. Protein expression levels were assessed by immunoblot analyses (Fig. 4a). Five clones were selected for further studies that all displayed an $80-100 \%$ reduction in gremlin 1 protein levels compared to cells harboring a non-targeting (NT) guide sequence. The gremlin1 depleted clones showed no consistent change in growth rate or ability to form colonies in soft agar 
A

\begin{tabular}{lrrrrr} 
& \multicolumn{2}{c}{ Cells } & & \multicolumn{2}{c}{ Tumors } \\
\cline { 2 - 3 } \cline { 5 - 6 } Bmper & 13,79 & 7,61 & 6,09 & 1,67 \\
Crim1 & 21,29 & 16,49 & & 12,55 & 12,52 \\
Dand5 & 2,18 & 3,66 & & 3,59 & 3,42 \\
Fst & 1,82 & 0,06 & 5,27 & 0,26 \\
Fstl1 & 4,91 & 0,06 & 50,75 & 9,67 \\
Grem1 & 15,47 & 0,00 & 6,12 & 0,01 \\
Nbl1 & 0,14 & 0,23 & 2,52 & 1,59 \\
Twsg1 & 18,04 & 26,45 & 34,96 & 47,86 \\
Smad6 & 2,92 & 0,99 & 2,74 & 0,79 \\
Smad7 & 11,21 & 5,72 & 9,52 & 4,24 \\
Smad4 & 25,25 & 22,84 & 35,26 & 29,75
\end{tabular}

C

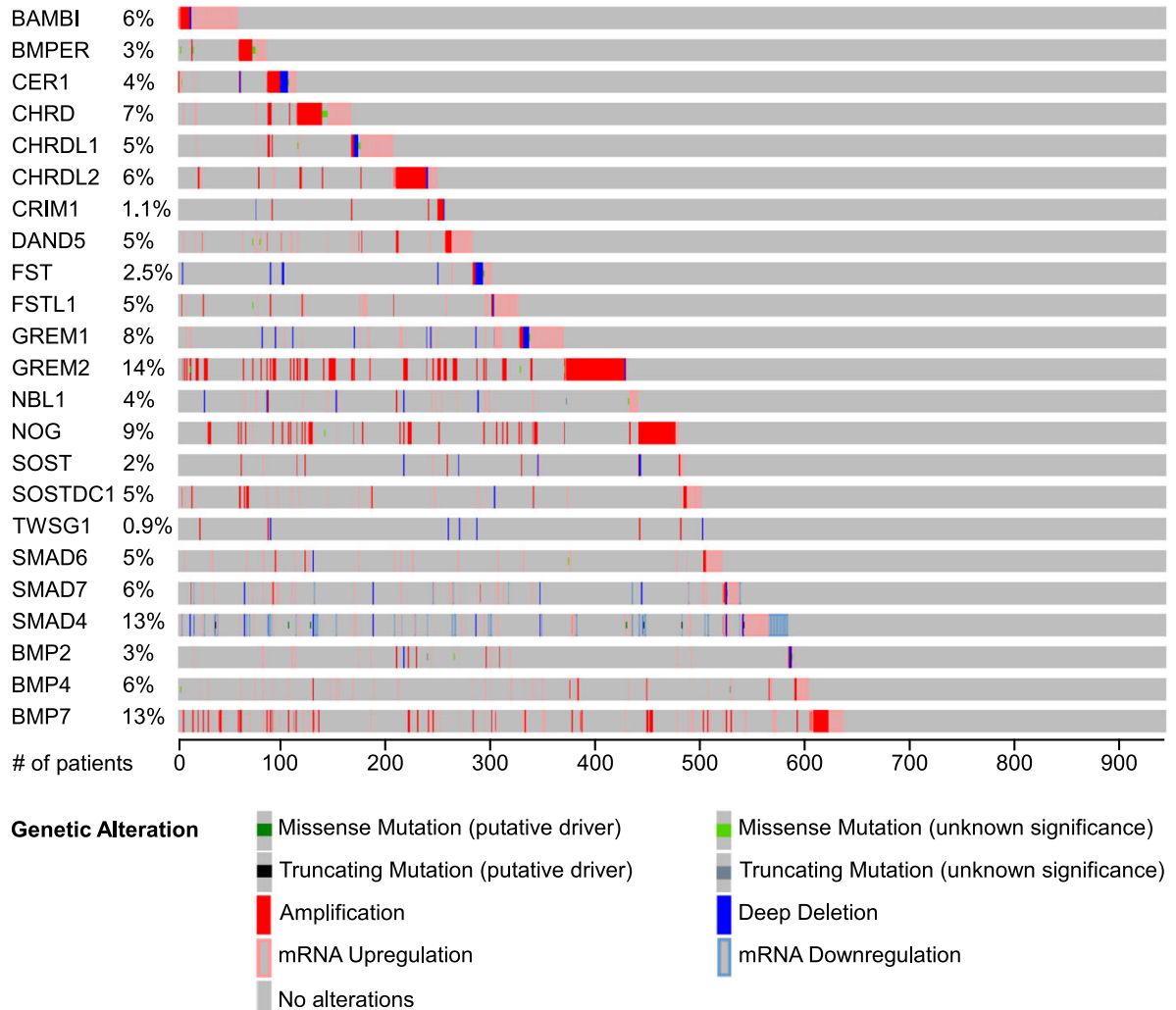

Fig. 2 BMP-antagonists are genetically altered in a high frequency of breast cancer biopsies. (a) Heat-map showing the RNA-seq expression levels of 11 BMP-antagonists (cut-off: expression level $\geq 1$ in either in vitro cultivated cells or tumors of 67NR and 66cl4). Values are given in fragments per kilobase of transcripts per million fragments mapped (FPKM). (b) Gene enrichment analysis of the 1252 genes significantly upregulated in 67NR cells and primary tumors using the Enrichr online tool (TRANSFAC and JASPAR position weight matrices). (c) Genetic alterations in extracellular, intracellular and transmembrane BMP-antagonists, as well as BMP2, BMP4 and BMP7 were analyzed using cBioPortal in 960 patient samples from The Cancer Genome Atlas (TCGA) provisional data set for invasive breast carcinoma

compared to the pooled 66cl4 NT control cells (Additional file 8: Figure S2A and B). Next, we analyzed the ability of two randomly selected clones to form primary tumors and lung metastases after injection into the fat pad and tail vein of nude mice, respectively. Unfortunately, none of the Cas9 expressing clones could form primary tumors in immunocompetent $\mathrm{BALB} / \mathrm{c}$ mice, irrespective of gene editing.
However, all cell lines formed tumors in immunocompromised, nude mice. Compared to control cells, the Grem1 depleted clones Grem1_1.3 and Grem1_2.1 formed smaller primary tumors and lost their ability to metastasize to the lungs (Fig. $4 \mathrm{~b}-\mathrm{d}$ ). In addition to the non-target cell line NT_mix, two control single cell clones (NT_1 and NT_2) were analyzed in vivo. Surprisingly, these two control sub-clones did not behave like 
A

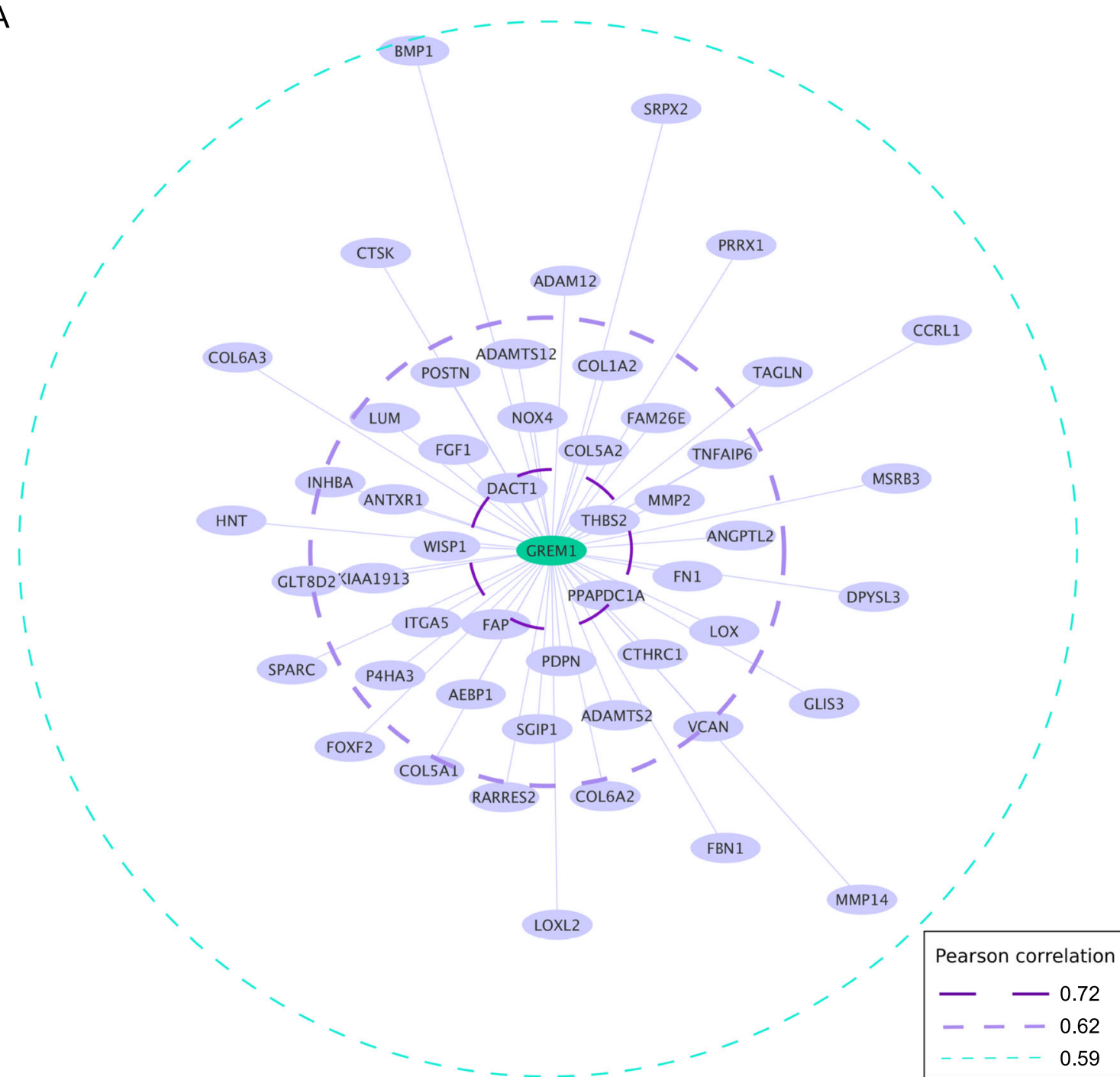

B

$\begin{array}{rrr} & \text { Combined score } & \text { P-value } \\ \text { Extracellular matrix organization_Homo sapiens_R-HSA-1474244 } & 99.35 & 3.614 \mathrm{e}-21 \\ \text { Collagen formation_Homo sapiens_R-HSA-1474290 } & 56.71 & 6.364 \mathrm{e}-13 \\ \text { Assembly of collagen fibrils and other multimeric structures_Homo sapiens_R-HSA-2022090 } & 56.07 & 8.084 \mathrm{e}-13 \\ \text { Collagen biosynthesis and modifying enzymes_Homo sapiens_R-HSA-1650814 } & 44.53 & 1.960 \mathrm{e}-10 \\ \text { Crosslinking of collagen fibrils_Homo sapiens_R-HSA-2243919 } & 26.74 & 1.222 \mathrm{e}-6 \\ \text { Elastic fibre formation_Homo sapiens_R-HSA-1566948 } & 23.98 & 3.269 \mathrm{e}-6 \\ \text { Diseases of glycosylation_Homo sapiens_R-HSA-3781865 } & 23.78 & 2.665 \mathrm{e}-6 \\ \text { Integrin cell surface interactions_Homo sapiens_R-HSA-216083 } & 20.61 & 2.359 \mathrm{e}-5 \\ \text { Axon guidance_Homo sapiens_R-HSA-422475 } & 18.38 & 2.759 \mathrm{e}-4 \\ \text { Activation of Matrix Metalloproteinases_Homo sapiens_R-HSA-1592389 } & 18.02 & 6.929 \mathrm{e}-5\end{array}$

Fig. 3 (See legend on next page.) 
(See figure on previous page.)

Fig. 3 GREM1 expression is associated with genes involved in collagen formation and extracellular matrix (ECM) organization. Co-expression analysis was done on RNA-seq data from 421 breast cancer cases downloaded from the TCGA (study by Ciriello et al., Cell, 2015) using Pearson correlation coefficient. (a) Depicts the 50-top scoring genes in an ego-centric network and (b) shows the results of the gene enrichment analysis of these genes using the Reactome pathway database

66cl4 wildtype or the NT_mix control cells, but rather displayed impaired primary tumor growth and metastases formation in the lungs similar to the gremlin 1 depleted clones. From this, it is apparent that sub-clones originating from single 66cl4 cells have different ability to form primary and secondary tumors without any further manipulation. Nonetheless, gremlin1 is overexpressed in the metastatic 66cl4 versus the non-metastatic 67NR cells. This, and the finding that GREM1 is frequently overexpressed in breast cancers and correlates with poor prognosis, merge on the notion that gremlin1 is important for aggressive breast cancer development.

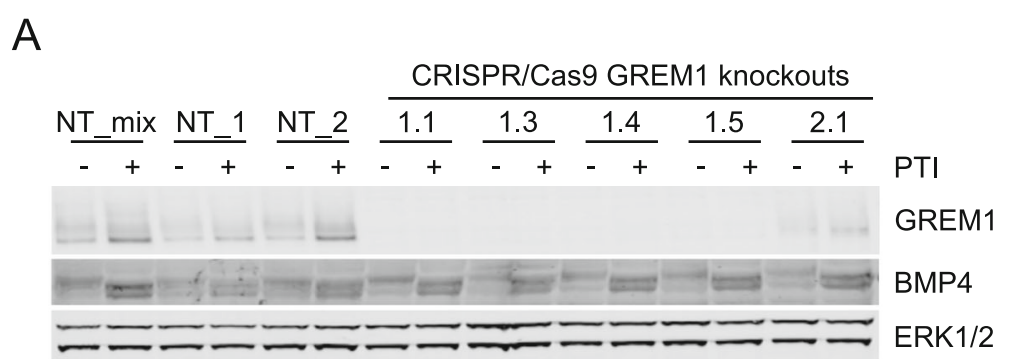

B

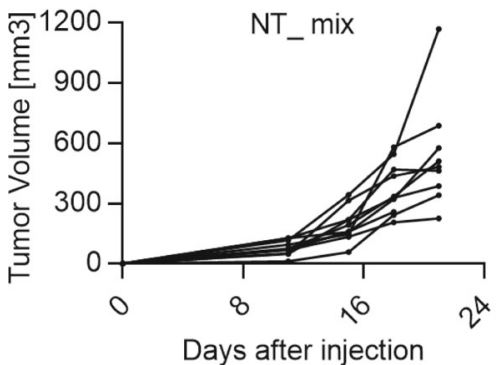

C

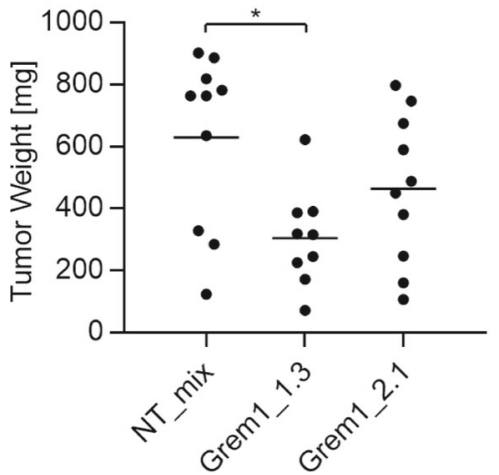

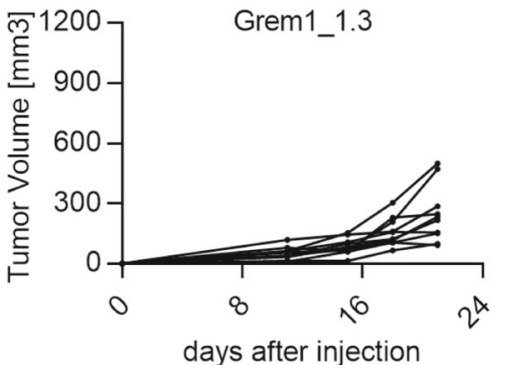

days after injection

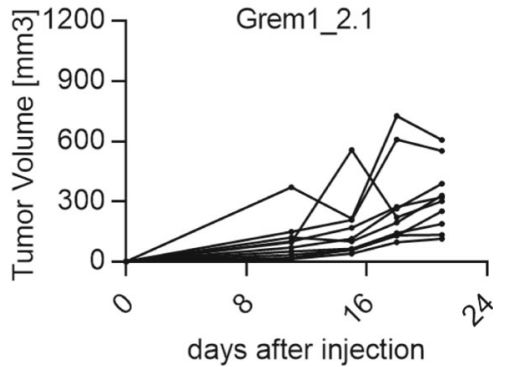

$\mathrm{D}$

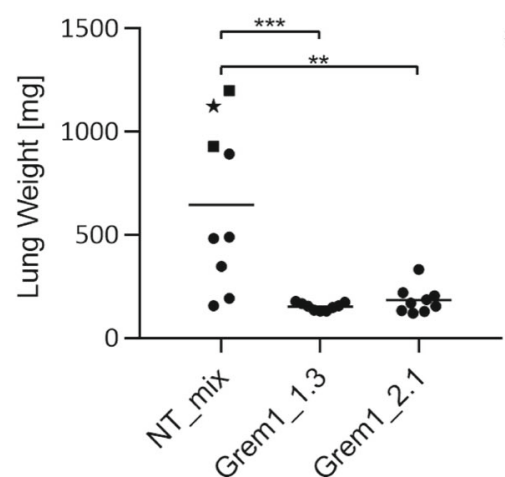

sacrificed earlier:

- 2 days

$\star 1$ day

Fig. 4 Gremlin1 depletion impairs metastases formation in the lungs. (a) Analysis of CRISPR/Cas9-mediated knockout efficiency in 66cl4. Representative immunoblot of gremlin 1 of whole lysates of $66 \mathrm{cl} 4$ non-target controls and gremlin 1 knockout clones. (b-c) In vivo analysis of $66 \mathrm{cl} 4$ non-target control and two gremlin1 knockout clones (10 mice per group). Tumor cells were injected into the fat-pad of nude mice. (b) The size of the primary tumors was measured regularly during the 21 days of experiment and (c) tumor weight was recorded after the mice had been sacrificed. The line indicates mean tumor weight. Student's t-test, ${ }^{*} 0.01<P<0.05$. (d) Tumor cells were injected into the tail vein of nude mice. After 15 days the mice were sacrificed and the lung weight was recorded. The line indicates mean lung weight. Student's t-test, ${ }^{* *} 0.001<P<0.01,{ }^{* * *} P<0.001$ 
66cl4 displayed increased expression of stem cell markers The first step in metastasis is invasion of tumor cells into the surrounding stroma. Cancer cells of epithelial origin acquire the ability to invade as a part of the EMT, during which they reverse to an undifferentiated, stem cell-like state [12]. Since BMP-antagonists, such as gremlin1, can interfere with differentiation, we were interested in the differentiation status of the metastatic 66cl4 and the nonmetastatic 67NR cells in the initial RNA-seq experiment. Analyzing the transcripts of 13 expressed stem cell markers, we found that four markers were significantly upregulated in 66cl4 cells and primary tumors $(C d 24 a$, Krt8, Itgb4 and Esr1), compared to 67NR cells and 67NR tumors (Fig. 5a and Additional file 9: Table S6). On the other side, none of the stem cell markers analyzed were significantly upregulated in $67 \mathrm{NR}$ compared to $66 \mathrm{cl} 4$. In breast cancer, a mesenchymal $\mathrm{CD} 44^{\text {hi }} \mathrm{CD} 24^{\text {low }}$ phenotype is suggested to characterize the cancer stem cells [34]. However, of the stem cell markers analyzed in 66cl4, $C d 24 a$ was the most clearly elevated. Flow cytometry analysis of CD24 expression on 66cl4 and 67NR in vitro cultured cells confirmed the results of the RNA-seq analysis (Fig. 5b). Cd44, on the other hand, showed significantly lower expression in 66cl4 compared to 67NR (Fig. 5a and Additional File 9: Table S6). Thus, the expression of these two markers indicate the opposite of expected for a classic stem cell-like metastatic phenotype $\left(\mathrm{CD} 44^{\mathrm{hi}} \mathrm{CD} 24^{\mathrm{low}}\right)$ in 66cl4. This is consistent with previous studies showing that the metastatic potential of the 4T1 cell line is much stronger than the non-metastatic 67NR, even though 4T1 is characterized by an epithelial phenotype and the 67NR cells are more differentiated towards a mesenchymal
A

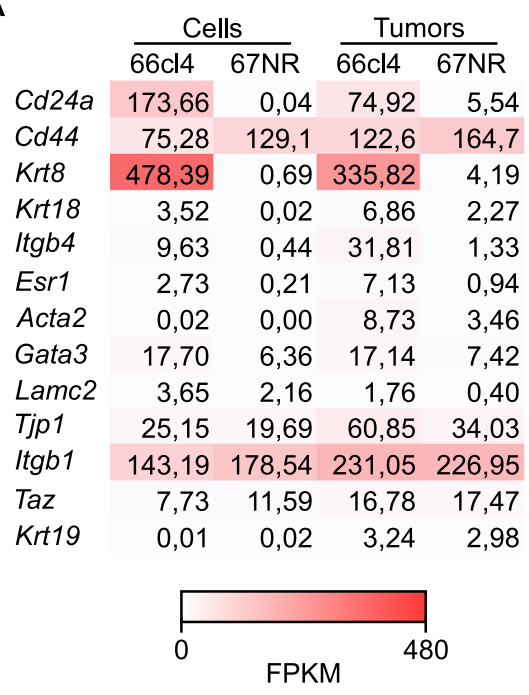

C

CD24

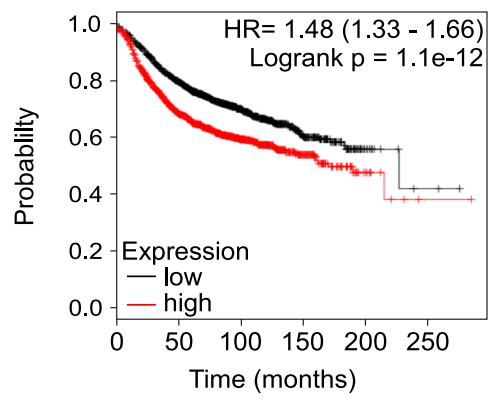

Number at risk

low $19761397 \quad 629 \quad 136 \quad 15 \quad 2$

high $19751122 \quad 446 \quad 105 \quad 12 \quad 1$
B

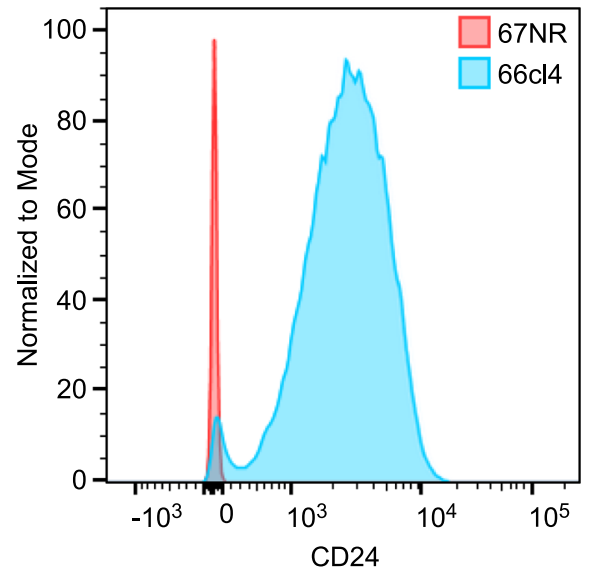

D

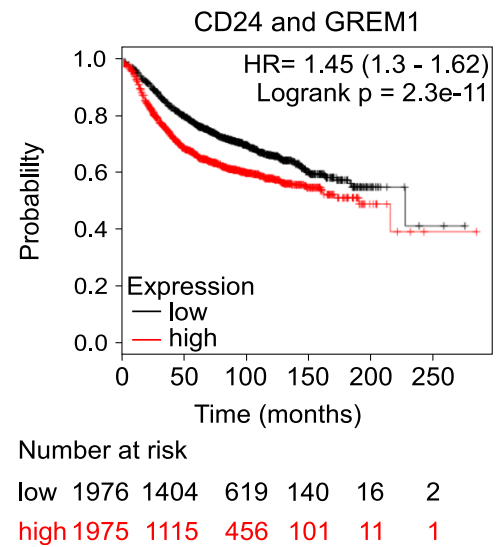

Fig. 5 High Grem 1 levels correlates with high expression of stem cell markers in 66cl4. (a) Heat-map showing the RNA-Seq expression levels of 13 known stem cell markers (cut-off: expression level $\geq 1$ in either cells or tumors of 67NR and 66cl4). Values are given in fragments per kilobase of transcripts per million fragments mapped (FPKM). (b) Representative histogram of 67NR and 66cl4 in vitro cultured cells stained with CD24 anti-mouse antibody. (c) Relationship between CD24 gene expression and RFS in breast cancer patients using KM plotter. (d) Relationship between combined CD24 and GREM1 expression and RFS in breast cancer patients using KM plotter 
phenotype [35]. It was also proposed that the 66cl4 cells display a poorer ability to invade and migrate in vitro compared to the 67NR cells, although 66cl4 metastasizes to lungs in vivo [36]. To get a better overview of the phenotypic profile, we used ChIP-X enrichment analysis (ChEA) in the Enrichr database to analyze the 1270 genes significantly upregulated in both 66cl4 cells and 66cl4 tumors. The analysis showed activation of several signaling pathways that are essential for stem cell maintenance, including Oct4, Sox2, Sox9, and Nanog (Additional file 10: Figure S3). Taken together, although the expression of commonly used breast cancer stem cell markers showed opposite expression than what would be expected, we found increased expression of several stem cell markers in 66cl4 compared to 67NR, supporting a metastatic stem cell-like phenotype. Since increased GREM1 mRNA expression levels in tumors correlated with reduced RFS, we asked if high CD24 mRNA levels also could predict poor prognosis. Similar to GREM1, CD24 correlated with reduced RFS in all breast cancer cases when highly expressed (Fig. 5c). Of note, combining the expression levels for GREM1 and CD24 did not give any additive effect in prediction value, suggesting that their functions are closely related (Fig. 5d).

\section{Extravasation capacity is only marginally affected by gremlin 1 depletion}

Since gremlin1 depletion might impair the metastatic potential of $66 \mathrm{cl} 4$, we next asked if $66 \mathrm{cl} 4$ and $67 \mathrm{NR}$ also display a different ability to extravasate and if gremlin1 has a role in controlling such differences. We first took advantage of an in vitro approach, where the cancer cells were scored for their ability to interfere with the integrity of a confluent layer of human umbilical vein endothelial cells (HUVECs) measured real time using impedance [37]. After seeding the HUVECs, the impedance increased until it reached a plateau. Visual inspection of the wells verified that the HUVECs had adhered and that the cultures were confluent. A small volume of culture medium containing the same number of $67 \mathrm{NR}$ or $66 \mathrm{cl} 4$ cells was added to the respective wells and impedance measured. The same volume of conditioned medium (CM) from the two cell lines was used as a negative control. Presence of 66cl4 cells caused a rapid loss in impedance (Fig. 6a). In contrast, adding the same number of 67NR cells resulted in a less prominent effect. These data suggest that the metastatic, gremlin1expressing 66cl4 cells are better in penetrating an endothelial layer than 67NR. Interestingly, we did not see any change in impedance when adding conditioned medium of $66 \mathrm{cl} 4$ and $67 \mathrm{NR}$ to the HUVEC monolayer (Fig. 6b), indicating that changes in the endothelial cell monolayer are caused by direct interactions of the tumor cells with the HUVECs.
To test if the impaired metastatic potential of $66 \mathrm{cl} 4$ GREM1_1.3 and GREM1_2.1 in vivo was due to a reduced capability to extravasate, we repeated the in vitro extravasation assay with these two gremlin 1 depleted cell lines. We found that both clones had a slightly reduced ability to penetrate the HUVEC monolayer compared to the NT control cells (Fig. 6c). In summary, these data suggest that the metastatic $66 \mathrm{cl} 4$ cells are more able to penetrate an endothelial layer than the non-metastatic 67NR cells. However, depletion of gremlin1 in 66cl4 only marginally affected the extravasation ability. As an alternative and more invasion relevant approach, we employed a xenograft model using the transgenic zebrafish line $\operatorname{Tg}(f l i 1: G F P)$ [16-18]. After injecting fluorescently labelled cancer cells in the duct of Cuvier (DoC) of zebrafish embryos at $48 \mathrm{~h}$ post fertilization, the movement of cancer cells into the avascular tail fin area, which is a measure for invasion, was documented using a fluorescent microscope. Consistent with the findings of the in vitro extravasation assay, 66cl4 wildtype cells and NT_mix cells displayed similar numbers of invasion clusters, whereas 66cl4 Grem1_1.3 formed fewer clusters in the avascular tail fin area (Fig. 6d and e).

\section{Co-expression of GREM1 and BMPs does not annul the prognostic value of GREM1 in ER-negative breast cancer patients}

The finding that Bmp4 and its antagonist Grem1 were coexpressed in the 66cl4 metastatic cell line puzzled us. We therefore looked for co-expression of GREM1 and its preferred BMPs, BMP2, BMP4 or BMP7, in human breast cancer cell lines using Expression Atlas [28]. Interestingly, all the 13 breast cancer cell lines that expressed GREM1 co-expressed at least one of the three BMPs, with BMP4 being most commonly co-expressed (Additional file 11: Figure S4). To explore the clinical significance of these findings, we used the TCGA provisional data set for invasive breast carcinoma in cBioPortal to analyze if BMPantagonists were co-expressed with either $B M P 2, B M P 4$, or $B M P 7$. We found that many of the biopsies with a BMP amplification, also had an amplification of a gene encoding one of the BMP-antagonists (Fig. 2c). For BMP7, several amplifications were also seen without a concomitant amplification of any known antagonist.

Since deregulated BMP activity has been linked to invasion and metastasis [38], we wanted to explore the clinical significance of elevated BMP levels for survival in breast cancer patients. However, we found no correlation between high expression level of $B M P 2, B M P 4$ or $B M P 7$ and prognosis, irrespective of sub-grouping (all breast cancer cases, ER-positive subtype, or ER-negative subtype (Table 1). Based on our findings that elevated BMP expression does not correlate with reduced RFS, whereas high GREM1 expression is associated with poor 


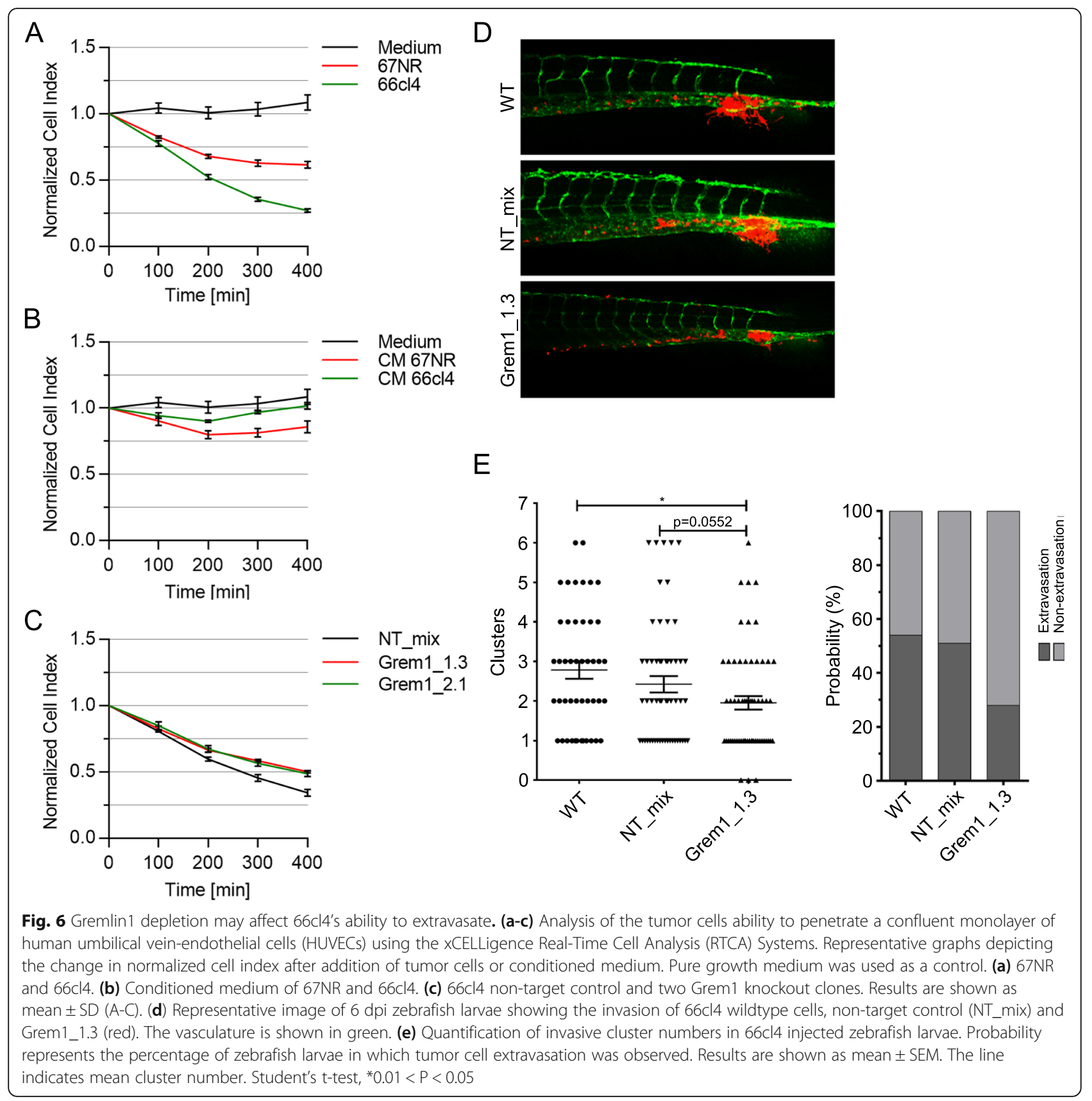

prognosis in breast cancer, we hypothesized that combining the expression levels of BMPs and GREM1 annuls the observed correlation of GREM1 with RFS. Surprisingly, this was not the case. We found that in ERnegative breast cancer cases combining high expression level of GREM1 with high expression of BMP2, BMP4 or $B M P 7$ showed a slightly poorer prognosis compared to GREM1 alone (Table 1). Combined, these data demonstrate that breast cancer cells may co-express BMPs and GREM1, and that co-expression of BMP and GREM1 in breast cancer biopsies does not reduce GREM1's value as a predictor of poor outcome.
Increased levels of GREM1 mRNA are associated with lack of estrogen receptor expression

The prognostic value of GREM1 in ER-negative breast cancers made us ask if there could be a link between GREM1 and ER-signaling. We therefore analyzed cell line data available from the Cancer Cell Line Encyclopedia (Broad Institute). We found that 10\% (6 out of 60) breast cancer cell lines expressed elevated GREM1 mRNA levels compared to the average of $>1000$ different cell lines (Fig. 7a). The expression of GREM1 mRNA was not caused by copy number alterations in the gene, and interestingly, all the GREM1 expressing 
Table 1 Combined elevated GREM1 and BMP expression level improves prognostic value in ER-negative breast cancer patients. Analysis of RFS in breast cancer patients using KM plotter. High and low expression were defined as above and below median

\begin{tabular}{|c|c|c|c|c|c|c|c|}
\hline \multirow[t]{2}{*}{ Gene(s) } & \multirow[t]{2}{*}{ Gene ID } & \multicolumn{2}{|l|}{ all BC patients } & \multicolumn{2}{|l|}{ ER+ BC patients } & \multicolumn{2}{|l|}{ ER- BC patients } \\
\hline & & $\mathrm{HR}$ & $p$-value & $\mathrm{HR}$ & $p$-value & $\mathrm{HR}$ & $p$-value \\
\hline GREM1 & 218469_at & $1.32(1.18-1.47)$ & $6.90 \mathrm{E}-07$ & $1.19(1.01-1.4)$ & 0.035 & $1.51(1.2-1.9)$ & 0.00037 \\
\hline BMP2 & 205289_at & $0.88(0.79-0.98)$ & 0.02 & $0.88(0.75-1.04)$ & 0.13 & $1.01(0.81-1.26)$ & 0.94 \\
\hline BMP4 & 211518_s_at & $0.9(0.81-1)$ & 0.058 & $0.98(0.83-1.16)$ & 0.83 & $1.16(0.93-1.46)$ & 0.19 \\
\hline$B M P 7$ & 209590_at & $0.88(0.79-0.98)$ & 0.023 & $1.08(0.92-1.27)$ & 0.36 & $0.92(0.73-1.15)$ & 0.45 \\
\hline BMP2/GREM1 & 205289_at / 218469_at & $1.33(1.19-1.48)$ & 3.70E-07 & $1.21(1.03-1.42)$ & 0.022 & $1.64(1.31-2.07)$ & $1.80 \mathrm{E}-\mathrm{C}$ \\
\hline BMP4/GREM1 & 211518_s_at / 218469_at & $1.28(1.15-1.43)$ & 7.30E-06 & $1.2(1.02-1.42)$ & 0.026 & $1.63(1.3-2.06)$ & $2.30 \mathrm{E}-05$ \\
\hline BMP7/GREM1 & 209590_at / 218469_at & $1.29(1.16-1.44)$ & 4.10E-06 & $1.19(1.01-1.4)$ & 0.04 & $1.63(1.3-2.06)$ & $2.30 \mathrm{E}-05$ \\
\hline
\end{tabular}

cell lines lacked expression of estrogen receptor, ESR1, mRNA (Fig. 7b). Moreover, in the TCGA provisional dataset (cBioportal) there was no overlap between breast cancer biopsies overexpressing GREM1 $(n=61)$ or ESR1 $(n=44)$ mRNA (data not shown), further supporting a functional link between GREM1 expression and lack of ER-signaling.

\section{Discussion}

Both activated BMP signaling [39-42] and elevated levels of SMAD-inhibitors [43, 44] and BMP-antagonists $[8-10,31,32]$ have been linked to tumor progression and metastasis. Moreover, amplification and/or upregulation of BMPs as well as BMP-antagonists, including NOG, GREM1, GREM2, and CHRD have been reported in breast cancer [39, 45]. Yet, the simultaneous upregulation of BMPs and their antagonists, and the fact that both promote tumor aggressiveness appears contradictory. In the present study, we found that high expression of the BMPantagonist GREM1 correlates strongly with reduced RFS in ER-negative breast cancer.
Analyzing the metastatic $66 \mathrm{cl} 4$ and non-metastatic $67 \mathrm{NR}$ cell lines of the 4T1 mouse mammary tumor model, we found that Grem 1 was upregulated in $66 \mathrm{cl} 4$ cells and primary tumors. Consistent with the role of gremlin1 as an antagonist of BMP-induced differentiation, RNA-seq revealed that $66 \mathrm{cl} 4$ cells have elevated expression of several stem cell markers compared to 67NR. In line with Goa et al. [8], who found that overexpression of Dand5 in the $4 \mathrm{TO} 7$ mouse mammary tumor cell line inhibits lung-derived BMPs and thereby promotes metastases in the lungs, we observed a reduction in lung metastases after injection of $66 \mathrm{cl} 4$ Grem1 depleted cells into the tail vein of nude mice. However, high levels of DAND5 in patients did not predict survival and may be less relevant than GREM1 in breast cancer development. Interestingly, of the BMP-antagonists tested here, only elevated expression of GREM1 and CRIM1 correlated with poor prognosis in patients with ER-negative tumors.

The clinical relevance of our findings in the 4T1 mouse mammary tumor model is supported by several observations. Most importantly, high GREM1 expression
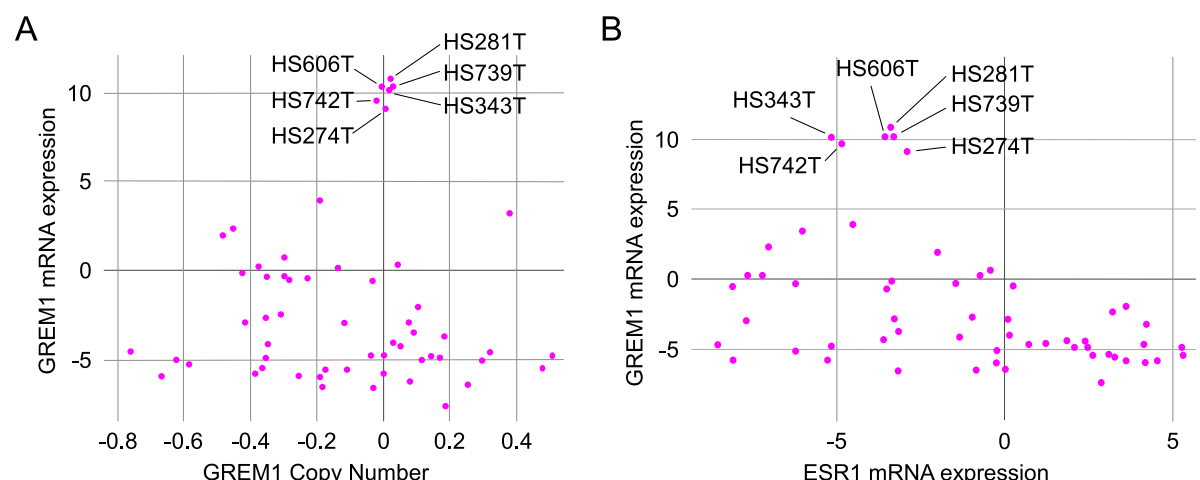

Fig. 7 Increased levels of GREM1 mRNA are associated with lack of estrogen receptor expression. Level of GREM1 transcript in 60 different human breast cancer cell lines analyzed using the Cancer Cell Line Encyclopedia made available by Broad Institute. (a) GREM1 transcript levels were elevated in some cell lines, but this did not correlate with GREM1 copy number variations. (b) Correlation of the GREM1 and estrogen receptor (ERS1) transcript levels. Transcript levels were determined using RNA-sequencing and compared to the average expression in more than 1000 human cancer cell lines for each transcript 
correlated with reduced relapse-free survival in ERnegative breast cancer patients. Using publicly available databases, we found that GREM1 is also expressed on mRNA level by various human breast cancer cell lines and that GREM1 is significantly upregulated in primary tumor biopsies of breast cancer patients compared to normal tissue samples. However, it remains unclear which cells are the source of GREM1 in the tumor biopsies. The gene alteration data (Fig. 2c) shows that GREM1 mRNA is elevated in approximately $6 \%$ of the breast cancer biopsies, and that amplifications are not common for this gene. It was recently shown that GREM1 can be produced by cancer-associated fibroblasts (CAFs) in breast cancer patients and that CAFs are the main source of GREM1 in colorectal cancer tissue $[46,47]$. Of note, GREM1 was one of eight elevated genes shown in cancer cells laser-dissected from invasive breast carcinoma patients compared with cancer cells from ductal carcinoma in situ patients [48], supporting that GREM1 could be expressed by the transformed cancer cells themselves and associated with an invasive phenotype. Taken together, the elevated GREM1 mRNA found in breast cancer biopsies could either come from the cancer cells themselves, or from cells infiltrating the tumor.

Among the 28 mRNAs encoding secreted proteins that were significantly upregulated in $66 \mathrm{cl} 4$ cells and tumors, we found not only Grem1, but also its ligand, Bmp4. Like other members of the TGF- $\beta$ superfamily, BMP4 has both tumor-suppressing and tumorpromoting roles in tumor cells [40]. On one side, it has been demonstrated that BMP4 inhibits tumor cell growth. On the other side, BMP4 has been shown to increase cell migration and invasion of tumor cells. Recently, it has been described that BMP4 might regulate autophagy [49] and polarize macrophages towards an anti-inflammatory or M2-like phenotype [50]. Using web-based databases we analyzed mRNA expression levels of GREM1 and BMP4 in breast cancer cell lines and a breast cancer patient cohort (TCGA) and found that these two genes are also co-expressed in some tumor cell lines and primary tumors. In this way, we confirmed a possible relevance of this unexpected coexpression of both BMP4 and its antagonist gremlin1 specifically in the aggressive 66cl4 cells.

In addition to BMP4, also elevated levels of other members of the BMP-family, including BMP6 [51, 52] and BMP7 [53], have been linked to increased tumor aggressiveness. Interestingly, in contrast to GREM1, we found no correlation between high mRNA expression level and poor prognosis for breast cancer patients for any of the three BMPs tested. However, the correlation between GREM1 expression levels and RFS in ERnegative breast cancer cases was not annulled when combining high expression levels of GREM1 and BMP2, $B M P 4$ or BMP7. These data indicate that gremlin1 and BMP may not counteract each other with respect to cancer progression. Since gremlin1 has been shown to bind to cell surfaces [54], one could speculate that gremlin1, secreted by tumor cells or surrounding cells, binds to the surface of the transformed cancer cells and protects them from BMP-induced differentiation. On the other hand, BMPs could be further distributed in the TME and thereby induce differentiation of immune cells and stroma cells to promote tumor growth and metastasis.

In this study, we show that GREM1 mRNA in tumor biopsies correlates with poor survival in ER-negative breast cancer patients. We propose that there may be an association between expression of GREM1 and lack of expression of the estrogen receptor, ESR1, as we only found GREM1 expression among the ESR1 negative tumor cells. Our findings are consistent with a previous study showing that BMPs can counteract estrogen-induced cell division and that estrogen treatment led to downregulation of several BMP-receptors, including BMPR1A [55]. BMPR1A is needed for proper BMP2 and BMP4 activity and downregulating this receptor could be one way that ER-positive breast cancer cells avoid BMP activity. We speculate that ERnegative cells may develop other mechanisms to avoid BMP activity, for instance by producing their own BMP antagonists or by modulating the microenvironment to secrete factors that inhibit BMPs. We also observed that $6 \%$ of the tumor biopsies had elevated GREM1 mRNA levels (Fig. 2c) as opposed to $10 \%$ of the breast cancer cell lines (Fig. 7). Since the source of GREM1 in some of the tumor biopsies likely are CAFs, the proportion of cell lines that make their own GREM1 is relatively large and this may indicate that GREM1 gives them a growth advantage that enrich for such cells among the breast cancer cell lines.

\section{Conclusions}

We find that gene expression of BMP-antagonists is more frequently elevated than expression of inhibitory SMADs in breast cancer biopsies. Elevated expression level of GREM1 in tumor biopsies correlate with adverse outcome irrespective of the expression level of the BMPs in the biopsy. This suggests a dominant role for gremlin1 secretion with respect to tumor progression. The predictive value of the gene expression level for the different BMP-antagonists varies and suggests that the antagonists are functionally different in ways that must be better understood to explore these as possible drug targets against metastatic breast cancer development. 


\section{Supplementary information}

Supplementary information accompanies this paper at https://doi.org/10. 1186/s12964-019-0467-7.

Additional file 1: Supplementary methods: Transcriptome analysis, Quantitative PCR, Immunoblotting, Conditioned medium, ELISA, Cell proliferation assay, Soft-agar assay, Flow cytometry, and In vitro extravasation assay using xCELLigence Real-Time Cell Analysis (RTCA) Systems.

Additional file 2: Figure S1. High GREM1 expression correlates with OS in breast cancer patients. Relationship between GREM1 gene expression and OS in breast cancer patients using KM plotter. High and low expression was determined using best cut off.

Additional file 3: Table S1. Genes encoding secreted proteins that are significantly upregulated in $66 \mathrm{cl} 4$ and their prognostic value in breast cancer patients. High and low expression were defined as above $(H R>1.2$, p-value $<0.05)$ and below $(H R<0.83$, p-value $<0.05)$ median.

Additional file 4: Table S2. RNA-Seq expression levels of BMPantagonists and SMADs. Expression level $\geq 1$ in either cells or tumors of 67NR and $66 \mathrm{cl}$. Values are given in fragments per kilobase of transcripts per million fragments mapped (FPKM), as well as Log2 and p-values.

Additional file 5: Table S3. Relationship between gene expression of BMP-antagonists and RFS in breast cancer patients. High and low expression were defined as above ( $\mathrm{HR}>1.2$, $\mathrm{p}$-value $<0.05)$ and below $(H R<0.83$, $p$-value < 0.05) median.

Additional file 6: Table S4. The 50 top-scoring genes that are coexpressed with GREM1 in breast cancer. Co-expression analysis of the 50 top-scoring hits that are found co-expressed with GREM1 in a search of 331 breast cancer data sets in the SEEK database.

Additional file 7: Table S5. GREM1 expression is associated with genes involved in extracellular matrix (ECM) and collagen fibril organization. Gene enrichment analysis (GO Biological Process (BP) terms) of 50 topscoring hits that co-expressed with GREM1 using the SEEK database. T, term size; A, Number of genes in the co-expressed gene set with annotations in the functional database; A\&T, size of overlap between the term's gene-set and the co-expressed gene set.

Additional file 8: Figure S2. In vitro analysis of CRISPR/Cas9-mediated Grem1 knockouts in $66 \mathrm{cl} 4$. (A) Measurement of proliferation in culture $(n=4)$. Results are shown as mean \pm SEM. Student's t-test, ${ }^{*} 0.01<P<0.05,{ }^{* * *} P<0.001$. (B) Soft-agar assay. Colony area was measured in pixels $(n=3)$. Results are shown as mean \pm SEM

Additional file 9: Table S6. RNA-Seq expression levels of 13 known stem cell markers. Expression level $\geq 1$ in either cells or tumors of $67 \mathrm{NR}$ and $66 \mathrm{cl}$. Values are given in fragments per kilobase of transcripts per million fragments mapped (FPKM), as well as Log2 and p-values.

Additional file 10: Figure S3. Signaling pathways maintaining stemness are activated in $66 \mathrm{cl} 4$. Using CHiP-X enrichment analysis (ChEA) of the 1,270 genes significantly upregulated in both $66 \mathrm{cl} 4$ cells and $66 \mathrm{cl} 4$ tumors, we found activation of several signaling pathways that are essential for stem cell maintenance.

Additional file 11: Figure S4. GREM1 is co-expressed with BMPs in several human breast cancer cell lines. Co-expression analysis of GREM1 and selected BMPs (BMP2, BMP4, and BMP7) in human breast cancer cell lines using Expression atlas.

\section{Abbreviations}

BMP: Bone morphogenetic protein; ChEA: ChIP-X enrichment analysis: CM: Conditioned medium; DoC: Duct of Cuvier; Dpi: Days post implantation; ECM: Extracellular matrix; EMT: Epithelial-to-mesenchymal transition; ER: Estrogen receptor; FPKM: Fragment per kilobase of mRNA per million mapped reads; GDF: Growth and differentiation factor; HR: Hazard ratio; HUVECs: human umbilical vein endothelial cells; NT: Non-targeting; OS: Overall survival; Q-PCR: Quantitative-PCR; RFS: Relapse free survival; RNAseq: RNA-sequencing; SD: Standard deviation; SEEK: Search-Based Exploration of Expression Compendium [Human]; SEM: Standard error of the mean; TCGA: The Cancer Genome Atlas; TGF- $\beta$ : transforming growth factor- $\beta$; TME: Tumor microenvironment

\section{Acknowledgements}

We thank Unni Nonstad for excellent technical assistance. The RNAsequencing was provided by the Genomics Core Facility (GCF), Norwegian University of Science and Technology (NTNU). GCF is funded by the Faculty of Medicine and Health Sciences at NTNU and Central Norway Regional Health Authority. We also thank the Comparative medicine Core Facility (CoMed), NTNU, for the help regarding the mice experiments. CoMed is funded by the Faculty of Medicine and Health Sciences at NTNU and Central Norway Regional Health Authority. Parts of the results shown here are based upon data generated by the TCGA Research Network: https://www.cancer. gov/tcga.

\section{Authors' contributions}

UN, CW, GB and TH prepared the manuscript, UN, CW, MH, EA, JR, GB, PtD and $\mathrm{TH}$ collected the data, UN, CW, MH, EA, JR, SZ, BJ, RIS, GB, PtD and TH analyzed the data, GB, PtD and TH did the conceptualization, UN, CW, MH, EA, JR, SZ, BJ, RIS, GB, PtD and TH edited the manuscript. All authors read and approved the final manuscript.

\section{Funding}

This work was supported by grants from the Norwegian Cancer Society (project numbers: 63843 and 63825), and from the Research Council of Norway through its Centres of Excellence funding program (project number: 223255/F50), by a grant from the Norwegian Women's Public Health Association (CW) and Cancer Genomics Center Netherlands (PtD).

\section{Availability of data and materials}

The transcriptome data obtained by sequencing mRNA isolated from cells and primary breast tumors of $67 \mathrm{NR}$ and $66 \mathrm{cl} 4$ is accessible from NCBI (https://www.ncbi.nlm.nih.gov/biosample, SRA accession PRJNA577616).

\section{Ethics approval}

The mice studies were approved by the National Animal Research Authorities and carried out according to the European Convention for the Protection of Vertebrates used for Scientific Purposes (FOTS ID 10049). The zebrafish experiment was approved by The Institutional Committee for Animal Welfare of the Leiden University Medical Center (LUMC). All experiments were performed according to approved guidelines and regulations.

\section{Consent for publication}

Not applicable.

\section{Competing interests}

The authors declare that they have no competing interests.

\section{Author details}

${ }^{1}$ Centre of Molecular Inflammation Research, Department of Clinical and Molecular Medicine, Faculty of Medicine and Health Sciences, NTNU Norwegian University of Science and Technology, Trondheim, Norway. ${ }^{2}$ Department of Biomedical Laboratory Science, Faculty of Natural Sciences, NTNU - Norwegian University of Science and Technology, Trondheim, Norway. ${ }^{3}$ Clinic of Laboratory Medicine, St Olavs Hospital, Trondheim, Norway. ${ }^{4}$ Department of Biotechnology and Food Science, Faculty of Natural Sciences, NTNU - Norwegian University of Science and Technology, Trondheim, Norway. ${ }^{5}$ K.G. Jebsen Center for Genetic Epidemiology, Department of Public Health and General Practice, Faculty of Medicine and Health Sciences, NTNU - Norwegian University of Science and Technology, Trondheim, Norway. ${ }^{6}$ Department of Cell and Chemical Biology, Oncode Institute, Leiden University Medical Center, Leiden, The Netherlands. ${ }^{7}$ Department of Molecular Oncology, Institute for Cancer Research, Oslo University Hospital-Radiumhospitalet, Oslo, Norway. ${ }^{8}$ Department of Informatics, University of Oslo, Oslo, Norway. ${ }^{9}$ Department of Clinical and Molecular Medicine (IKOM), NTNU, Gastro Center, Prinsesse Kristinas gt 1, 7030 Trondheim, Norway. ${ }^{10}$ Department of Hematology, St. Olavs Hospital, Trondheim, Norway. 
Received: 16 July 2019 Accepted: 22 October 2019

Published online: 06 November 2019

\section{References}

1. Wakefield LM, Hill CS. Beyond TGF $\beta$ : roles of other TGF $\beta$ superfamily members in cancer. Nat Rev Cancer. 2013;13(5):328-41.

2. Colak S, Ten Dijke P. Targeting TGF- $\beta$ signaling in Cancer. Trends in cancer 2017;3(1):56-71.

3. Tang B, Vu M, Booker T, Santner SJ, Miller FR, Anver MR, Wakefield LM. TGF$\beta$ switches from tumor suppressor to prometastatic factor in a model of breast cancer progression. J Clin Invest. 2003;112(7):1116-24.

4. Mu Y, Gudey SK, Landstrom M. Non-Smad signaling pathways. Cell Tissue Res. 2012;347(1):11-20

5. Zhang L, Ye Y, Long X, Xiao P, Ren X, Yu J. BMP signaling and its paradoxical effects in tumorigenesis and dissemination. Oncotarget. 2016; 7(47):78206-18.

6. Avsian-Kretchmer O, Hsueh AJ. Comparative genomic analysis of the eightmembered ring cystine knot-containing bone morphogenetic protein antagonists. Molecular endocrinology (Baltimore, Md). 2004;18(1):1-12.

7. Walsh DW, Godson C, Brazil DP, Martin F. Extracellular BMP-antagonist regulation in development and disease: tied up in knots. Trends Cell Biol. 2010;20(5):244-56

8. Gao H, Chakraborty G, Lee-Lim AP, Mo Q, Decker M, Vonica A, Shen R, Brogi E, Brivanlou AH, Giancotti FG. The BMP inhibitor coco reactivates breast cancer cells at lung metastatic sites. Cell. 2012;150(4):764-79.

9. Yan K, Wu Q, Yan DH, Lee CH, Rahim N, Tritschler I, DeVecchio J, Kalady MF, Hjelmeland AB, Rich JN. Glioma cancer stem cells secrete Gremlin1 to promote their maintenance within the tumor hierarchy. Genes Dev. 2014; 28(10):1085-100.

10. Davis H, Irshad S, Bansal M, Rafferty H, Boitsova T, Bardella $C$, Jaeger E, Lewis A, Freeman-Mills L, Giner FC, et al. Aberrant epithelial GREM1 expression initiates colonic tumorigenesis from cells outside the stem cell niche. Nat Med. 2015;21(1):62-70

11. Hanahan D, Weinberg RA. Hallmarks of cancer: the next generation. Cell. 2011;144(5):646-74.

12. Batlle E, Clevers H. Cancer stem cells revisited. Nat Med. 2017;23(10):1124-34.

13. Dexter DL, Kowalski HM, Blazar BA, Fligiel Z, Vogel R, Heppner GH. Heterogeneity of tumor cells from a single mouse mammary tumor. Cancer Res. 1978;38(10):3174-81.

14. Miller FR, Miller BE, Heppner GH. Characterization of metastatic heterogeneity among subpopulations of a single mouse mammary tumor: heterogeneity in phenotypic stability. Invasion \& metastasis. 1983;3(1):22-31.

15. Church RH, Krishnakumar A, Urbanek A, Geschwindner S, Meneely J, Bianchi A, Basta B, Monaghan S, Elliot C, Stromstedt M, et al. Gremlin1 preferentially binds to bone morphogenetic protein-2 (BMP-2) and BMP-4 over BMP-7. The Biochemical journal. 2015:466(1):55-68.

16. Lawson ND, Weinstein BM. In vivo imaging of embryonic vascular development using transgenic zebrafish. Dev Biol. 2002;248(2):307-18.

17. He S, Lamers GE, Beenakker JW, Cui C, Ghotra VP, Danen EH, Meijer AH, Spaink HP, Snaar-Jagalska BE. Neutrophil-mediated experimental metastasis is enhanced by VEGFR inhibition in a zebrafish xenograft model. J Pathol. 2012;227(4):431-45.

18. Ren J, Liu S, Cui C, Ten Dijke P. Invasive behavior of human breast Cancer cells in embryonic Zebrafish. Journal of visualized experiments : JoVE. 2017:122.

19. Ciriello G, Gatza ML, Beck AH, Wilkerson MD, Rhie SK, Pastore A, Zhang H, McLellan M, Yau C, Kandoth C, et al. Comprehensive molecular portraits of invasive lobular breast Cancer. Cell. 2015;163(2):506-19.

20. Gyorffy B, Lanczky A, Eklund AC, Denkert C, Budczies J, Li Q, Szallasi Z. An online survival analysis tool to rapidly assess the effect of 22,277 genes on breast cancer prognosis using microarray data of 1,809 patients. Breast Cancer Res Treat. 2010;123(3):725-31.

21. Nagy A, Lanczky A, Menyhart O, Gyorffy B. Validation of miRNA prognostic power in hepatocellular carcinoma using expression data of independent datasets. Sci Rep. 2018;8(1):9227.

22. Gao J, Aksoy BA, Dogrusoz U, Dresdner G, Gross B, Sumer SO, Sun Y, Jacobsen A, Sinha R, Larsson E et al: Integrative analysis of complex cancer genomics and clinical profiles using the cBioPortal. Sci Signal 2013, 6(269):pl1

23. Cerami E, Gao J, Dogrusoz U, Gross BE, Sumer SO, Aksoy BA, Jacobsen A Byrne CJ, Heuer ML, Larsson E, et al. The cBio cancer genomics portal: an open platform for exploring multidimensional cancer genomics data. Cancer discovery. 2012;2(5):401-4.

24. Chen EY, Tan CM, Kou Y, Duan Q, Wang Z, Meirelles GV, Clark NR, Ma'ayan A. Enrichr: interactive and collaborative HTML5 gene list enrichment analysis tool. BMC bioinformatics. 2013;14:128.

25. Kuleshov MV, Jones MR, Rouillard AD, Fernandez NF, Duan Q, Wang Z, Koplev S, Jenkins SL, Jagodnik KM, Lachmann A, et al. Enrichr: a comprehensive gene set enrichment analysis web server 2016 update. Nucleic Acids Res. 2016;44(W1):W90-7.

26. Fabregat A, Jupe S, Matthews L, Sidiropoulos K, Gillespie M, Garapati P, Haw $R$, Jassal B, Korninger F, May B, et al. The Reactome pathway knowledgebase. Nucleic Acids Res. 2018;46(D1):D649-d655.

27. Zhu Q, Wong AK, Krishnan A, Aure MR, Tadych A, Zhang R, Corney DC, Greene CS, Bongo LA, Kristensen VN et al: Targeted exploration and analysis of large cross-platform human transcriptomic compendia. Nature methods 2015, 12(3):211-214, 213 p following 214.

28. Petryszak R, Keays M, Tang YA, Fonseca NA, Barrera E, Burdett T, Fullgrabe A, Fuentes AM, Jupp S, Koskinen S, et al. Expression atlas update--an integrated database of gene and protein expression in humans, animals and plants. Nucleic Acids Res. 2016;44(D1):D746-52.

29. Ghandi M, Huang FW, Jane-Valbuena J, Kryukov GV, Lo CC, McDonald ER 3rd, Barretina J, Gelfand ET, Bielski CM, Li H, et al. Next-generation characterization of the Cancer cell line encyclopedia. Nature. 2019;569(7757):503-8.

30. Naba A, Clauser KR, Hoersch S, Liu H, Carr SA, Hynes RO. The matrisome: in silico definition and in vivo characterization by proteomics of normal and tumor extracellular matrices. Molecular \& cellular proteomics : MCP. 2012; 11(4):M111.014647.

31. Karagiannis GS, Musrap N, Saraon P, Treacy A, Schaeffer DF, Kirsch R, Riddell $\mathrm{RH}$, Diamandis EP. Bone morphogenetic protein antagonist gremlin-1 regulates colon cancer progression. Biol Chem. 2015;396(2):163-83.

32. Hsu MY, Rovinsky SA, Lai CY, Qasem S, Liu X, How J, Engelhardt JF, Murphy GF. Aggressive melanoma cells escape from BMP7-mediated autocrine growth inhibition through coordinated Noggin upregulation. Laboratory investigation; a journal of technical methods and pathology. 2008;88(8):842-55.

33. Wilkinson L, Kolle G, Wen D, Piper M, Scott J, Little M. CRIM1 regulates the rate of processing and delivery of bone morphogenetic proteins to the cell surface. J Biol Chem. 2003:278(36):34181-8.

34. Lamouille S, Xu J, Derynck R. Molecular mechanisms of epithelialmesenchymal transition. Nat Rev Mol Cell Biol. 2014;15(3):178-96.

35. Marsan M, Van den Eynden G, Limame R, Neven P, Hauspy J, Van Dam PA, Vergote I, Dirix LY, Vermeulen PB, Van Laere SJ. A core invasiveness gene signature reflects epithelial-to-mesenchymal transition but not metastatic potential in breast cancer cell lines and tissue samples. PLoS One. 2014;9(2):e89262.

36. Lou Y, Preobrazhenska O, Auf dem Keller U, Sutcliffe M, Barclay L, PC MD, Roskelley C, Overall CM, Dedhar S. Epithelial-mesenchymal transition (EMT) is not sufficient for spontaneous murine breast cancer metastasis. Developmental dynamics : an official publication of the American Association of Anatomists. 2008;237(10):2755-68.

37. Rahim S, Uren A. A real-time electrical impedance based technique to measure invasion of endothelial cell monolayer by cancer cells. Journal of visualized experiments : JoVE. 2011;50.

38. Ren J, Ten Dijke P. Bone Morphogenetic Proteins in the Initiation and Progression of Breast Cancer. In: Vukicevic S, Sampath KT, editors. Bone Morphogenetic Proteins: Systems Biology Regulators: Cham: Springer International Publishing; 2017. p. 409-33.

39. Owens P, Pickup MW, Novitskiy SV, Giltnane JM, Gorska AE, Hopkins CR, Hong CC, Moses HL. Inhibition of BMP signaling suppresses metastasis in mammary cancer. Oncogene. 2015;34(19):2437-49.

40. Ketolainen JM, Alarmo EL, Tuominen VJ, Kallioniemi A. Parallel inhibition of cell growth and induction of cell migration and invasion in breast cancer cells by bone morphogenetic protein 4. Breast Cancer Res Treat. 2010; 124(2):377-86.

41. Alarmo EL, Parssinen J, Ketolainen JM, Savinainen K, Karhu R, Kallioniemi A. BMP7 influences proliferation, migration, and invasion of breast cancer cells. Cancer Lett. 2009;275(1):35-43.

42. Owens P, Polikowsky H, Pickup MW, Gorska AE, Jovanovic B, Shaw AK, Novitskiy SV, Hong CC, Moses HL. Bone morphogenetic proteins stimulate mammary fibroblasts to promote mammary carcinoma cell invasion. PLoS One. 2013;8(6):e67533. 
43. de Boeck M, Cui C, Mulder AA, Jost CR, Ikeno S, Ten Dijke P. Smad6 determines BMP-regulated invasive behaviour of breast cancer cells in a zebrafish xenograft model. Sci Rep. 2016;6:24968.

44. Stolfi C, Marafini I, De Simone V, Pallone F, Monteleone G. The dual role of Smad7 in the control of cancer growth and metastasis. Int J Mol Sci. 2013; 14(12):23774-90

45. Tarragona M, Pavlovic M, Arnal-Estape A, Urosevic J, Morales M, Guiu M, Planet E, Gonzalez-Suarez E, Gomis RR. Identification of NOG as a specific breast cancer bone metastasis-supporting gene. J Biol Chem. 2012;287(25):21346-55.

46. Dutton LR, Hoare OP, McCorry AMB, Redmond KL, Adam NE, Canamara S, Bingham V, Mullan PB, Lawler M, Dunne PD, et al. Fibroblast-derived Gremlin1 localises to epithelial cells at the base of the intestinal crypt. Oncotarget. 2019;10(45):4630-9.

47. Ren J, Smid M, laria J, Salvatori DCF, van Dam H, Zhu HJ, Martens JWM, Ten Dijke P. Cancer-associated fibroblast-derived gremlin 1 promotes breast cancer progression. Breast cancer research BCR. 2019;21(1):109.

48. Schultz S, Bartsch H, Sotlar K, Petat-Dutter K, Bonin M, Kahlert S, Harbeck N, Vogel U, Seeger H, Fehm T, et al. Progression-specific genes identified in microdissected formalin-fixed and paraffin-embedded tissue containing matched ductal carcinoma in situ and invasive ductal breast cancers. BMC Med Genet. 2018;11(1):80.

49. Deng G, Zeng S, Qu Y, Luo Q, Guo C, Yin L, Han Y, Li Y, Cai C, Fu Y, et al. BMP4 promotes hepatocellular carcinoma proliferation by autophagy activation through JNK1-mediated BCl-2 phosphorylation. Journal of experimental \& clinical cancer research : CR. 2018;37(1):156.

50. Martinez VG, Rubio C, Martinez-Fernandez M, Segovia C, Lopez-Calderon F, Garin MI, Teijeira A, Munera-Maravilla E, Varas A, Sacedon R, et al. BMP4 induces M2 macrophage polarization and favors tumor progression in bladder Cancer. Clinical cancer research : an official journal of the American Association for Cancer Research. 2017;23(23):7388-99.

51. Stieglitz D, Lamm S, Braig S, Feuerer L, Kuphal S, Dietrich P, Arndt S, Echtenacher B, Hellerbrand C, Karrer S, et al. BMP6-induced modulation of the tumor micro-milieu. Oncogene. 2018.

52. Darby S, Cross SS, Brown NJ, Hamdy FC, Robson CN. BMP-6 over-expression in prostate cancer is associated with increased id-1 protein and a more invasive phenotype. J Pathol. 2008;214(3):394-404.

53. Alarmo EL, Korhonen T, Kuukasjärvi T, Huhtala H, Holli K, Kallioniemi A. Bone morphogenetic protein 7 expression associates with bone metastasis in breast carcinomas. Ann Oncol. 2008;19(2):308-14.

54. Costello CM, Cahill E, Martin F, Gaine S, McLoughlin P. Role of gremlin in the lung: development and disease. Am J Respir Cell Mol Biol. 2010; 42(5):517-23.

55. Takahashi M, Otsuka F, Miyoshi T, Otani H, Goto J, Yamashita M, Ogura T, Makino H, Doihara H. Bone morphogenetic protein 6 (BMP6) and BMP7 inhibit estrogen-induced proliferation of breast cancer cells by suppressing p38 mitogen-activated protein kinase activation. J Endocrinol. 2008;199(3):445-55.

\section{Publisher's Note}

Springer Nature remains neutral with regard to jurisdictional claims in published maps and institutional affiliations.

Ready to submit your research? Choose BMC and benefit from:

- fast, convenient online submission

- thorough peer review by experienced researchers in your field

- rapid publication on acceptance

- support for research data, including large and complex data types

- gold Open Access which fosters wider collaboration and increased citations

- maximum visibility for your research: over $100 \mathrm{M}$ website views per year

At $\mathrm{BMC}$, research is always in progress.

Learn more biomedcentral.com/submissions 